\title{
Analysis of bookshelf mechanisms using the mechanics of Cosserat generalized continua
}

\author{
Rodrigo P. de Figueiredo ${ }^{\mathrm{a}}$, Eurípedes do A. Vargas $\mathrm{Jr}^{\mathrm{b}, *}$, Anderson Moraes ${ }^{\mathrm{c}}$ \\ ${ }^{a}$ Department of Mining Engineering of the Ouro Preto School of Mines, Federal University of Ouro Preto, Ouro Preto, Brazil \\ ${ }^{\mathrm{b}}$ Department of Civil Engineering, Catholic University of Rio de Janeiro and Geosciences Institute of Federal University of Rio de Janeiro, \\ Rio de Janeiro, RJ 22453-060, Brazil \\ ${ }^{\mathrm{c}}$ PETROBRAS_Petróleo Brasileiro SA, CENPES, Rio de Janeiro, Brazil
}

Received 25 August 2002; received in revised form 26 February 2004; accepted 3 March 2004

Available online 21 July 2004

\begin{abstract}
Bookshelf type mechanisms are commonly used to explain antithetic accommodation of deformations caused by direct shear. Stress analysis of such mechanisms have been proposed, mainly through the use of classic Mohr circle graphical constructions. The present work introduces an alternative framework for analysis, the mechanics of generalized Cosserat continua, although maintaining the same tools (Mohr circle construction). In this type of mechanics, kinematics of the material points incorporates independent rotational degrees of freedom besides the classic translational ones. In the equilibrium equations, quantities involving moments are generated and the stress tensor is in the general case non-symmetric. Such characteristics of generalized Cosserat continua make it suitable for the analysis of bookshelf mechanisms, as these are, in essence, rotational antithetic mechanisms induced by shear stresses having different magnitudes in perpendicular planes. The paper applies the mechanics of generalized continua to the analysis of a specific bookshelf mechanism, related to crustal extension. In the Appendices, a review of the generalized Cosserat continua and details of the graphical Mohr circle representation of stresses for such media are presented.
\end{abstract}

(C) 2004 Elsevier Ltd. All rights reserved.

Keywords: Bookshelf mechanism; Cosserat generalized continuum mechanics; Stress analysis; Mohr circles

\section{Introduction}

The analogy with a bookshelf type mechanism (Fig. 1) to describe the antithetic accommodation of deformation provoked by direct shear, or quasi-simple shear (Mandl, 2000), was initially proposed by Mandl (1987). Differently from the ideal simple shear, in direct shear, the parallel rock boundaries, where a constant normal stress is applied, are allowed to dilate or contract perpendicularly to the shear direction. Mandl (1987) analyzed the stress conditions that would lead to the above mentioned mechanism using conventional graphical representation of Mohr circles. Subsequently, Mandl (1988) presented a number of natural examples where the mechanism would be applicable. Recently, Mandl (2000) extended the discussion of the problem by giving it a role in the formation and analysis of other structures such as shear joints, parallel faults produced

\footnotetext{
* Corresponding author.

E-mail address: vargas@civ.puc-rio.br (E.A. Vargas).
}

by horizontal extension and inversion of normal and reverse faults.

Mandl (2000) distinguished two possible styles of accommodation of deformation by the proposed bookshelf mechanism (Fig. 1). The domino style is associated with an extension parallel to the direction of shear and with a contraction perpendicular to it. The dilational style, in contrast, has a contraction in the shear direction and an extension normal to this direction. In the domino style, the formation of antithetic $\mathrm{R}^{\prime}$ (Riedel) faults occurs, whereas in the dilational style, faults known as $\mathrm{P}^{\prime}$ type are formed (also antithetic, Fig. 1).

Independent of style, the analogy, as originally proposed, corresponds to a bookshelf with books, vertically disposed, above which a horizontal rigid board is placed (Fig. 1). Horizontal displacement of the board could in principle simulate the effect of shear on a block located between parallel faults or even strata of different competence on the limb of a flexural slip fold (Mandl, 1988). 


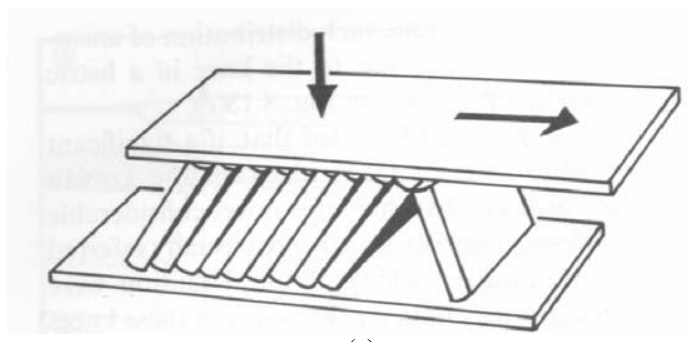

(a)

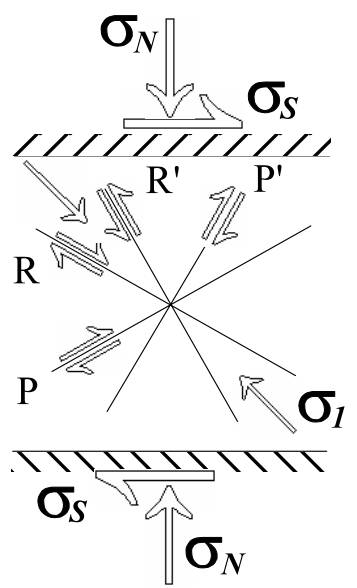

(b)

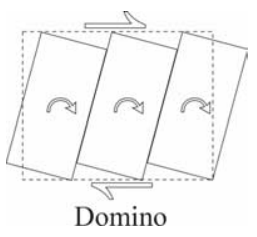

(c)

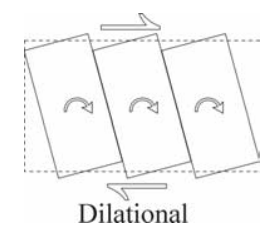

Fig. 1. (a) Analogy for the bookshelf mechanism (Mandl, 1988); (b) direct shear; (c) domino and dilational styles.

Fig. 1 shows that the direction given to the displacement of the upper board is not followed by the direction of sliding between the books. In fact, they rotate, an essential feature in order to accommodate the deformations. The surfaces of contact between the books represent the parallel antithetic faults. The books represent the blocks (lithons) separated by the faults. The analogy, in this sense, may appear rather exaggerated, as the books are rigid whereas real rock blocks are relatively more deformable. This, however, does not render it invalid qualitatively. The presence of rotations in the mechanism can only be explained if one assumes the existence of different values for shear stresses in the vertical and horizontal planes, which will then give rise to non equilibrated resultant moments. However, it is well known from classical mechanics that shear stresses in perpendicular planes are of equal magnitude (Cauchy stresses principle) and, as a consequence, the stress tensor is symmetric (Mase and Mase, 1992).

The authors argue therefore that one cannot interpret, in a theoretically consistent way, the bookshelf mechanism by using classic continuum mechanics, as it presupposes equal shear stresses in perpendicular planes, a fact that is incompatible with rotations. However, the generalized continuum mechanics of Cosserat (Cosserat and Cosserat, 1909) provides the necessary features to analyze the problem:

(a) independent rotational degrees of freedom in addition to the classic translational ones (see Appendix A, item A1);

(b) shear stresses on perpendicular planes which are not necessarily equal, but which are equilibrated through the introduction of new quantities, the so-called moment (or couple) stresses (see Appendix A, item A2).

As a consequence, parameters with dimensions of length appear in the stress-strain relationships and/or strength criteria. This fact brings with it a scale dependency effect, which is a well-known factor for geological materials in general (Jaeger and Cook, 1979).

The pioneer work systematizing the generalized mechanics was made in France by Cosserat and Cosserat (1909). The idea of an extension of the classic continuum, one which incorporated independent rotational degrees of freedom, as well as non symmetric shear stresses and couple stresses, had already been suggested in the nineteenth century by important theoreticians in continuum mechanics (Poisson, 1842; Cauchy, 1850; Saint Venant, 1869; Voigt, 1887). The theory remained practically forgotten until the mid sixties when it was reexamined, receiving a great theoretical impulse (Kröner, 1968). The number of foreseen applications was, however, relatively limited. One of the main suggestions at that time came from Mindlin (1963), for studies on stress concentrations around voids.

Lippmann (1969) pioneered the use of the theory to describe yielding/failure of granular materials. Chappell (1979), amongst others, proposed its use to describe the mechanics of fractured media. The repercussion of the latter work came years later through a publication by Besdo (1985). In these works, a correct modelling of the mechanical behaviour requires that the material points have independent rotational degrees of freedom. This is in fact, what distinguishes the kinematics of the Cosserat generalized mechanics from the classical mechanics (Appendix A, item A1).

In the 1980s, a growing interest in generalized mechanics occurred, starting with the work of Mühlhaus and Vardoulakis (1987), who proposed applications of the theory to the modelling of localization of plastic deformations in brittle materials. This problem is dependent on the scale of the microstructure (grains) and a clear independence was demonstrated between the rotations in the interior of the localization zone and the rotations outside this zone.

In relation to applications in geology, it appears that Biot 
(1967) was the first to use the Cosserat mechanics. Biot studied the problem of buckling of multilaminated, anisotropic rocks (e.g. Price and Cosgrove, 1990) and its relation with formation of folds and, in particular, of kink bands. His results, when using classic continuum, related the critical load causing folding to a zero fold wave length, which is, clearly, a physical inconsistency of the mathematical theory. This inconsistency could be corrected through the introduction of the Cosserat mechanics whereby the rock laminae were assigned thickness and flexural strength.

This work presents an analysis of the stresses involved in the bookshelf mechanism by using the graphical construction of the Mohr circle, as it has been done in the literature (see Mandl, 1987; 1988, 2000). However, the approach used differs from previous workers in that use is made of the mechanics of Cosserat generalized continua, which is thought to be a more adequate, if not a necessary basis, for the correct treatment of the problem. A description of a specific geological problem is presented, which is then followed by an analysis using Mohr circles adapted for Cosserat generalized continua. The Appendices give the basic elements for the understanding of the mechanics of Cosserat generalized continua and the adaptation of Mohr circles for such media.

\section{The problem}

The problem to be analyzed here (Fig. 2) is a version of the bookshelf mechanism that occurs under conditions of crustal extension at the surface. This problem was introduced by Ramsay and Huber (1987), from where Fig. 2 was extracted.

The decision to analyze this specific case instead of the direct shear cases treated by Mandl is because it has a well defined boundary condition: the free surface, represented by a horizontal plane, is the Earth's surface, where the tractions are identically zero. Such a boundary condition, with values known for both stress components, normal and shear, does not exist in the analyses presented by Mandl.

The stress conditions at a point on the surface are illustrated on Fig. 3. One can notice that in a horizontal plane, $\sigma_{\mathrm{V}}=\sigma_{\mathrm{HV}}=0$, where $\sigma_{\mathrm{V}}$ is the normal stress component (vertical) and $\sigma_{\mathrm{HV}}$ the shear stress component. On a vertical plane, $\sigma_{\mathrm{V}}$ and $\sigma_{\mathrm{VH}}$ are not necessarily zero. It should be noted that $\sigma_{\mathrm{HV}} \neq \sigma_{\mathrm{VH}}$ is mechanically sound when using the Cosserat generalized mechanics (see Appendix A).

In the adopted notation, the first subscript for the stresses represents the direction and, the second, the plane of action of the component. Such ordering of subscripts is contrary to what is generally found in texts dedicated to the stress analysis in classical continua (Jaeger and Cook, 1979). The reason for this is that the order becomes relevant as a function of the asymmetry of the stresses in the Cosserat generalized continua. The ordering appears as a consequence of theoretical consistency when, in the process of derivation, energy methods such as the Principle of Virtual Work (Appendix A, item A3) are used (Germain, 1973; Figueiredo, 1999).

A schematic illustration of the rigid blocks (assumed to be rectangular) formed by antithetic faults is shown on Fig. 3. Their dimensions are represented by a base $b$ and a height $h$. The introduction of block dimensions to the problem is another distinctive feature of the generalized mechanics in relation to the classic mechanics. In the example, the block dimensions (and shape) will make it possible to take into consideration an additional strength mechanism, besides the conventional Mohr-Coulomb one, which is related to the friction between blocks (Jaeger and Cook, 1979).

This mechanism involves 'rolling friction' (Nascimento and Teixeira, 1971; Fadeev and Kuzevanov, 1993) and is related to the toppling (rotation) of the blocks. The equation describing it is (see Appendix C):

$\sigma_{\mathrm{S}}=\sigma_{\mathrm{N}} \tan \varphi$,

where $\sigma_{\mathrm{S}}$ is the shear stress, $\sigma_{\mathrm{N}}$ is the normal stress and $\varphi=\tan ^{-1}(b / h)$ is the 'rolling friction' angle.

Eq. (1) provides, therefore, the value of the horizontal component of shear necessary to topple (rotate) a rectangular block, having width $b$ and height $h$, subjected to a normal vertical stress $\sigma_{\mathrm{N}}$. It has a similar form to the classical Mohr-Coulomb equation describing the shear strength:

$\sigma_{\mathrm{S}}=\sigma_{\mathrm{N}} \tan \phi$

where $\phi$ is the classical friction angle and the remaining symbols have the same meaning as in Eq. (1).

Additionally, a cohesive term could be added to both Eqs. (1) and (2). Furthermore, due to the non-existence of friction under tension (Jaeger and Cook, 1979), it is usual to add a tension cut-off to Eq. (2):

$\sigma_{\mathrm{N}} \leq T$

where $T$ is the tensile strength of the medium.

\section{Graphical stress analysis}

The basic difference introduced by the Cosserat generalized formulation in relation to the graphical 2D representation, given by the Mohr's circle, is the fact that the circle can have its center located away from the normal stress axis. This is because shear stresses in perpendicular directions (plotted as diametrically opposite points on the circle) may have different magnitudes. As a consequence, the principal planes will be not necessarily perpendicular (and therefore not necessarily represented by diametrically opposite points). In addition, principal stresses will no longer be the maximum and minimum normal stress components at a point (Appendix B).

Regarding the analyzed problem, one can assume, in principle, that before failure, the medium can be considered as approximately homogeneous and, therefore, behaving as a classical continuum. This fact does not alter the final 

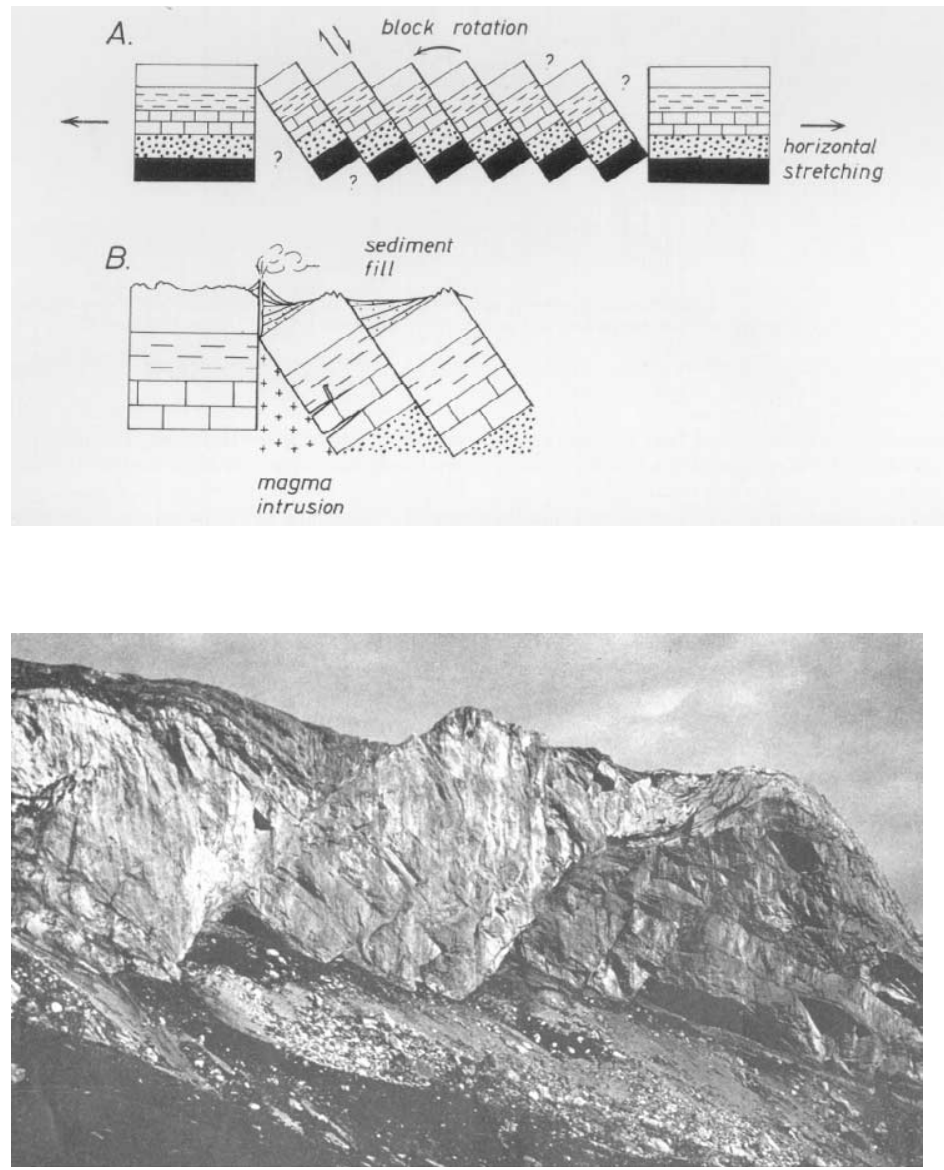

(c)

Fig. 2. Bookshelf mechanism in crustal extension (Ramsay and Huber, 1987): (a) and (b) mechanism; (c) illustration of an outcrop.

conclusions of the analysis (as will be explained in the sequence). It ultimately means that up to this point in the analysis it is not necessary to consider any intrinsic structure of the material. Such structure will appear later due to faulting.

In this case, vertical and horizontal planes will be initially the principal planes, and a Mohr circle for a point on the surface would be plotted with its center on the normal stress axis (circle 'a' in Fig. 4), as usual. Because this Mohr circle has its center on the normal stress axis, the shear stresses of diametrically located points, which correspond to the stresses on two perpendicular planes, are equal.

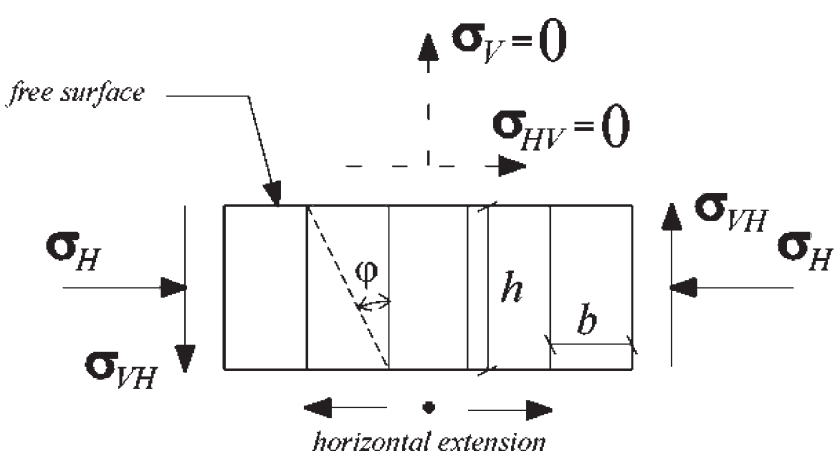

Fig. 3. State of stress for a point on the surface.
Fig. 4 shows the failure conditions through sliding, using the classical Mohr-Coulomb strength envelope (with a tension cut-off; Eq. (3)) together with a strength envelope for toppling (rotation) given by the strength criterion for rolling friction (represented by Eq. (1) with a cohesive term added). The crustal extension at the point analyzed can be represented in the Mohr space by a progressive reduction of $\sigma_{\mathrm{H}}$ (hypothetically assumed to be initially compressive) and

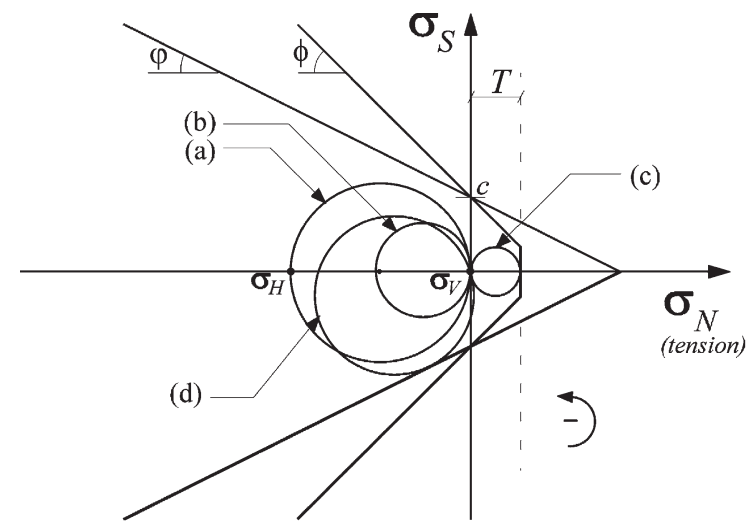

Fig. 4. Mohr circles and strength envelopes for analysis of the bookshelf problem under crustal extension (explanation of the various Mohr circles is given in the text). 
by maintaining the stress vector on the horizontal plane (free surface) equal to zero. This means that Mohr circles always pass through the origin.

If the medium behaved as a classical continuum, the point would be taken initially to the state of stresses represented by circle (b). Failure occurs when the circle touches the tensile strength envelope, as does circle (c), and would generate vertical failure planes. For the stress path followed, there is no possibility of shear failure of the Mohr-Coulomb type. This is because the diameter of the circle decreases constantly whilst the principal stresses are compressive. The diameter of the circle is only able to increase under tension where such criterion is no longer valid because of the absence of friction, already pointed out previously (Jaeger and Cook, 1979). Therefore, if it is accepted that the medium behaves as a classical continuum, the only explanation for the occurrence of failure by extension on the Earth's surface is the presence of tensile stresses. The occurrence of such tensile stresses, however, has not been supported either by experimental rock mechanics (e.g. Amadei and Stephansson, 1997) or by theoretical models (Sheorey, 1994). In addition, it is not possible to explain the observed in-situ rotations.

There remains therefore no other alternative but to abandon the axiom of symmetry in the classical stress tensor and to allow $\sigma_{\mathrm{VH}} \neq 0$, positive in the case of Fig. 4 and having the effect of a counter-clockwise couple (the sign convention for the Mohr circle is presented in Appendix B). At this stage, the framework of the generalized mechanics of Cosserat becomes convenient as it incorporates asymmetric shear stresses. Additionally, the other distinctive features of this mechanics (mentioned in Section 2) are also present. The internal structure represented by the blocks and its independent rotations are clearly noticed. In the Cosserat generalized mechanics, this structure will imply the existence of an intrinsic scale, which appears as characteristic lengths in the stress-strain relations and strength criteria, as shown in Appendixes A (item A4) and C, respectively.

It should be noted that $\sigma_{\mathrm{HV}}$ is zero due to the boundary condition (free surface), and that $\sigma_{\mathrm{VH}}=-2 \sigma^{\mathrm{a}}$, where $\sigma^{\mathrm{a}}=$ $\left(\sigma_{\mathrm{HV}}-\sigma_{\mathrm{VH}}\right) / 2 . \sigma^{\mathrm{a}}$ is the so-called anti-symmetric stress, one that quantifies the difference between shear stresses on perpendicular planes (see Appendix A). This implies that the circle, according to the Cosserat theory, will translate perpendicularly to the normal stress axis a distance equal to half $\sigma_{\mathrm{VH}}\left(\sigma_{\mathrm{VH}} / 2=\left|\sigma^{\mathrm{a}}\right|\right)$, in the sense of negative $\sigma_{\mathrm{S}}$. This leads to the situation represented by circle (d), which satisfies the boundary condition at the free surface, similarly to the previous classical stress circles. Circle (d) is able to displace, though always passing through the origin (because $\sigma_{\mathrm{V}}=0$ ), until it touches the Mohr-Coulomb envelope. However, it seems that blocks, generated by antithetic faults, can become unstable before this occurs, as shear failure surface, having the classic $\pm(\pi / 4-\phi / 2)$ inclination to the vertical, are not generally observed in these situations. In fact, failure may occur when circle (d) reaches the rolling friction envelope having an inclination angle $\varphi$ (see Section 2).

In Fig. 4, the Mohr-Coulomb envelope is plotted with a friction angle of $45^{\circ}$. This value should not be considered high as it is known that sliding can be very dilatant for low levels of normal stress (e.g. Goodman, 1976). In contrast, the rolling friction angle is given a slightly exaggerated value of $35^{\circ}$, so that the respective envelope plots inside to the MohrCoulomb envelope for compressive normal stresses. For this rolling friction angle, the blocks in Fig. 3 have a width/height ratio of 0.7 . More slender blocks could be generated by this antithetic failure mechanism, corresponding to lower values of $\varphi$. Therefore, failure could be even more likely than implied by Fig. 3 (because the respective envelope would lie below what was illustrated there). Thus, by admitting the existence of an a priori, intrinsic structure for the medium, the possibility of a rotational failure (toppling of blocks) becomes plausible as an alternative mechanism. This type of rotational failure causes the appearance of associated faults (in this case antithetic), in the same way as conventional faults are produced by simple shear.

It is remarkable that for an antithetic failure to occur, a minimal decrease in $\sigma_{\mathrm{H}}$ is required, in contrast to the classical failure mode where all planes but the horizontal must be under tensile stresses. Also, it is not necessary for a contraction of the circle to occur, as failure can be reached through its simple vertical translation. In the present analysis, a decrease of $\sigma_{\mathrm{H}}$ was adopted, consistent with crustal extension, which is evident from plate tectonics studies (e.g. Mattauer, 1973).

There are very few planes under tension as a result of the proposed mechanism and even those that do experience tensile stresses, when compared with the classical values, are of very small magnitude. The only way to generate classical shear failure would be to increase the vertical stress, concomitant with horizontal extension. This is clearly not possible in the case under analysis, as a point on the surface is being considered. As a result $\sigma_{\mathrm{V}}$ remains equal to zero.

It could be argued that faults are nucleated in the crust at depth, i.e. with a positive value of $\sigma_{\mathrm{V}}$. This would make the classical shear mechanism more plausible, but this depth factor in no way excludes the possibility of an antithetic faulting, as demonstrated in several seismic profiles of sedimentary basins (e.g. Mandl, 1988). Geological evidence of the type shown in Fig. 2b, however, given by recent sediments accumulated in sub-basins between toppled blocks, seem to contradict the possibility that the antithetic faults were formed in depth (e.g. on the limbs of a flexural slip fold), subsequently being brought to the surface by denudation.

\section{Conclusions and suggestions for additional applications}

The present work analyzes the bookshelf mechanism by using Mohr circles for a generalized Cosserat continuum. 
This analysis takes into consideration distinct strength envelopes for the two possible failure mechanisms; one involving rotation (antithetic faulting) and the other sliding. The main features of the theory of the generalized Cosserat continuum have been described with particular reference to those pertinent to the problem of the bookshelf mechanism.

The bookshelf mechanism can be interpreted in a simple way by using the mechanics of generalized Cosserat continua. By using this theory that takes into account rotations and the anti-symmetric portion of the stress tensor $\sigma^{\mathrm{a}}$, it is possible to explain the formation of antithetic faults. This type of faulting is related to rolling friction, which implies a lower strength when compared with sliding related faulting. By using the Cosserat theory it is not necessary to appeal for the unlikely tensile stresses in practically all planes, as required for classical failure mechanisms. Furthermore, through the use of Mohr circles and a Cosserat continuum, the analyses provides a way to incorporate rotations and $\sigma^{\mathrm{a}}$ (i.e. $\left.\left(\sigma_{\mathrm{HV}}-\sigma_{\mathrm{VH}}\right) / 2\right)$, both of which are responsible for the development of rotational antithetic faults. This is not possible when using the classical continuum, which does not incorporate these quantities.

Several problems in structural geology can be investigated under the framework of the theory of Cosserat generalized continua. An example is the problem of folding of multilaminated media as presented initially by Biot (1967) and complemented by Latham (1985a,b). Other problems, all of them involving rotations, include the analysis of the simple shear tectonics and its anastomosed patterns (in particular, the genesis of Riedel type fractures, $\mathrm{R}$ and $\mathrm{R}^{\prime}$ ), sigmoidal veins (Figueiredo and Vargas, 2001), the formation of kinkbands, etc. This paper aimed to demonstrate that Cosserat mechanics is not only appropriate for analysis of the bookshelf mechanism, but also for all geological processes that involve rotational antithetic mechanisms of deformation.

\section{Acknowledgements}

Part of this research work was carried out during the preparation of a Ph.D. thesis by the first author. PICDCAPES-UFOP/Brazil is acknowledged for financial support. J. Cosgrove, an anonymous reviewer and the editor J. Hippertt are thanked for their valuable comments and suggestions.

\section{Appendix A. The mechanics of 2D Cosserat generalized continua}

In its classic description, a continuum is a continuous distribution of particles, which are represented geometrically by a material point having cartesian coordinates $x_{i}$ $(i=1-3)$, with reference to a fixed system of orthogonal axes and characterized kinematically by a displacement vector $u_{i}$. In contrast, in the generalized continuum description, each point is seen as a particle having small dimensions, which is itself a small continuum around that point (Fig. A1). The kinematical characterization of the point or of the material particle strictu sensu, requires a refinement in relation to the classical continuum. The complexity of this refinement gives rise to the differences between the various generalized theories that have been proposed (Kröner, 1968).

In the Cosserat theory, the pioneer amongst the generalized theories, the material particle is rigid and has microrotations $\omega_{i}^{c}$ as additional degrees of freedom to $u_{i}$. This way, in such a continuum, the particle will have the degrees of freedom of a rigid body positioned in $x_{i}$. The gradients of microrotations, named curvatures, are added to the classical components of strain constituting a nonsymmetric tensor as shown in item A2 of this Appendix.

Through the Principle of Virtual Work (Chou and Pagano, 1967; Germain, 1973), it is possible to conjugate energetically the kinematical measures of the generalized continuum to their corresponding statical quantities, as briefly discussed in item A3 of this Appendix. As a result, the couple stress tensor, which is conjugate to the curvatures, and the force stress tensor of Cosserat, analogous to the classic stress tensor (although nonsymmetric), can be obtained. This asymmetry is particularly important to explain the bookshelf mechanism.

With the above mentioned static-kinematic refinements, the Cosserat theory allows representation of flexural and torsional effects, which are not present in the classic continuum. In contrast, the dimensions of the material particle, here denominated characteristic length, introduce a dependence on its underlying basic structure in the constitutive description (Jaeger and Cook, 1979).

In the particular case of rock masses, the rigid material

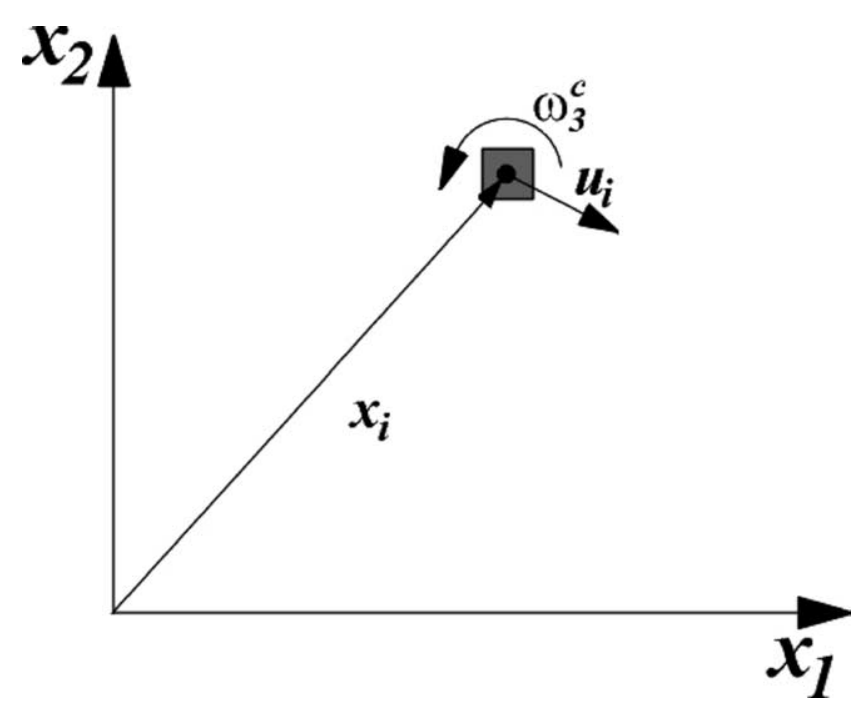

Fig. A1. Degrees of freedom for a material point in a Cosserat generalized continuum. 
particles of the Cosserat continua are able to represent the blocks formed between pre-existing geological discontinuities. This is so because these blocks are in general more rigid than the entire rock mass, where the deformability is concentrated on the interfaces (Goodman, 1976). In the sequence, a brief description of the kinematics of a $2 \mathrm{D}$ Cosserat continuum, restricted to small strains, is presented. A few remarks will be made about the constitutive relations for an isotropic linear elastic material and pertinent strength criteria.

\section{A1. Kinematics}

Consider a plan of deformations parallel to Cartesian orthogonal axes $\left(x_{1}, x_{2}\right) \cdot x_{i}(i=1,2)$ are the coordinates of a material point in a $2 \mathrm{D}$ Cosserat continuum. A rigid microparticle is associated with each point and, in the center of mass of the particle, a new local rigid cartesian coordinate system is located. During the deformation process, each particle moves $u_{i}$ and may suffer a rotation $\omega_{3}^{c}$ (Fig. A1). The subscript indicates that the rotation occurs around the axis $x_{3}$, normal to the plan of deformations, and the superscript is used to distinguish the Cosserat microrotations from the conventional macrorotations, which are in principle independent. This fact is crucial for the development of Cosserat theory, where the rotations bear no interdependence with the translational degrees of freedom, as occurs with the macrorotations of the classic mechanics.

The strain quantities in a Cosserat continuum are $\gamma_{i j}$ and $\kappa_{i}$, named, respectively, 'relative strains' (non-dimensional) and 'curvatures' (with dimensions of inverse of length), which are defined in 2D as:

$$
\begin{array}{ll}
\gamma_{11}=\partial_{1} u_{1}, & \gamma_{12}=\partial_{2} u_{1}+\omega_{3}^{c} ; \\
\gamma_{22}=\partial_{2} u_{2}, & \gamma_{21}=\partial_{1} u_{2}-\omega_{3}^{c} ; \\
\kappa_{1}=\partial_{1} \omega_{3}^{c}, & \kappa_{2}=\partial_{2} \omega_{3}^{c}
\end{array}
$$

where $\partial_{i}(\cdot)$ represents a gradient of $(\cdot)$ in the direction of $i$.

One can demonstrate (Figueiredo, 1999) that these quantities are objective, i.e. they are invariant with respect to rigid body movements, and can be used in the formulation of constitutive laws (Mase and Mase, 1992). As $\gamma_{12} \neq \gamma_{21}$, it is noticed that $\gamma_{i j}$ is non-symmetric in the non-trivial case where $\omega_{3}^{c} \neq 0$. It should be noted also that the symmetric part of $\gamma_{i j}$, $\gamma_{(i j)}=\left(\gamma_{i j}+\gamma_{j i}\right) / 2$, coincides with the classic strain tensor, $\epsilon_{i j}$, while its anti-symmetric part $\gamma_{[i j]}=\left(\gamma_{i j}-\gamma_{j i}\right) / 2$, is the difference between the macrorotations, $\Omega_{12}=\omega_{3}=\left(\partial_{2} u_{1}-\right.$ $\left.\partial_{1} u_{2}\right) / 2$, and the Cosserat microrotations $\omega_{3}^{c}$ (i.e. $\left.\gamma_{[21]}=\omega_{3}-\omega_{3}^{c}\right)$. An illustration of the kinematics represented by the expression of $\gamma_{12}$ is shown in Fig. A2.

The particular case, in which the Cosserat microrotations coincide with the rigid body macrorotations, i.e. when $\omega_{3}=$ $\omega_{3}^{c}$, was considered by Mindlin (1963) and used by Biot
(1967). This case is important only where the medium remains elastic. In the plastic domain, micro and macrorotations can be significantly different (Cosserat and Cosserat, 1909; Mühlhaus and Vardoulakis, 1987), as is generally the case in structural geology and particularly in the analysis of bookshelf mechanisms.

\section{A2. Statics}

A 2D Cosserat continuum contains six static quantities, energetically conjugate (through the Principle of Virtual Work) to the kinematic quantities defined in item A1. Therefore, besides the conventional stress tensor $\sigma_{i j}$, hereafter denominated force stress tensor, there are two additional components of couple (or moment) stresses $m_{i}=$ $m_{3 i}(i=1,2)$, with dimensions of moment by units of area.

In a plan element of infinitesimal dimensions $d x_{1}$ and $d x_{2}$ parallel to the coordinate axes (Fig. A3), it is assumed that an average value can be adopted for the distribution of force stress vectors and couple stresses on their faces. Moreover, it is also possible to neglect the terms higher than the first order, in a Taylor series approximation of the stress components on a face $x_{i}+d x_{i}$, as function of those on face $x_{i}$. By stating the equilibrium of forces according to the directions $x_{1}$ and $x_{2}$, the following relationships between force stresses in a Cosserat continuum are obtained:

$\partial_{1} \sigma_{11}+\partial_{2} \sigma_{12}+F_{1}=0$

$\partial_{1} \sigma_{21}+\partial_{2} \sigma_{22}+F_{2}=0$

where $F_{i}$ are body forces. In analogy, by stating the moment equilibrium in relation to the central point of the element

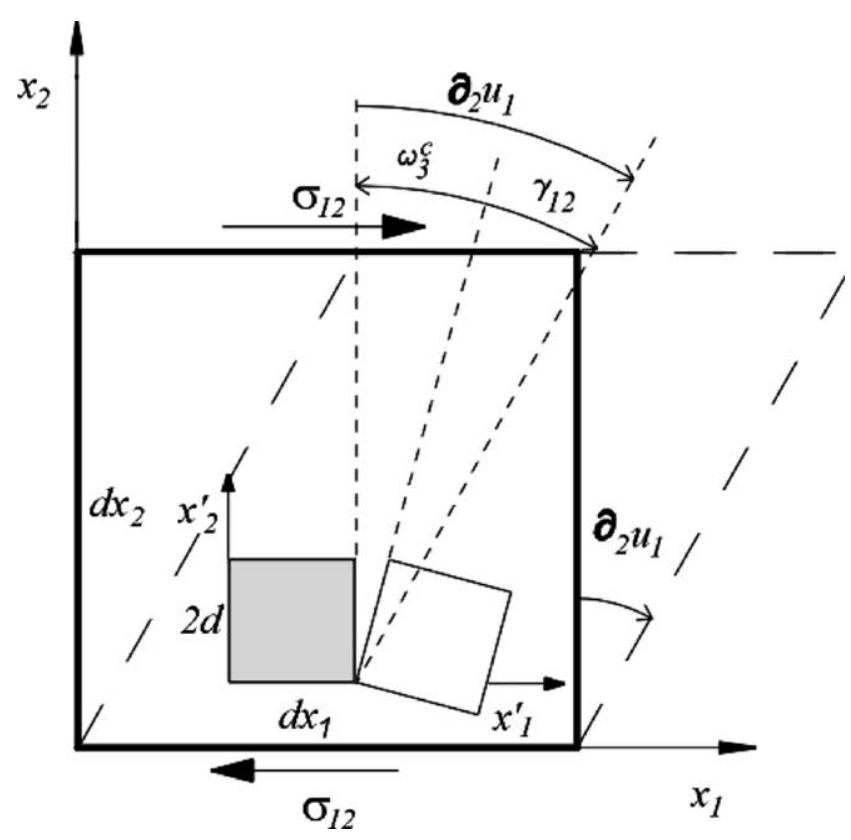

Fig. A2. Kinematic relationships for $\gamma_{12}$. 

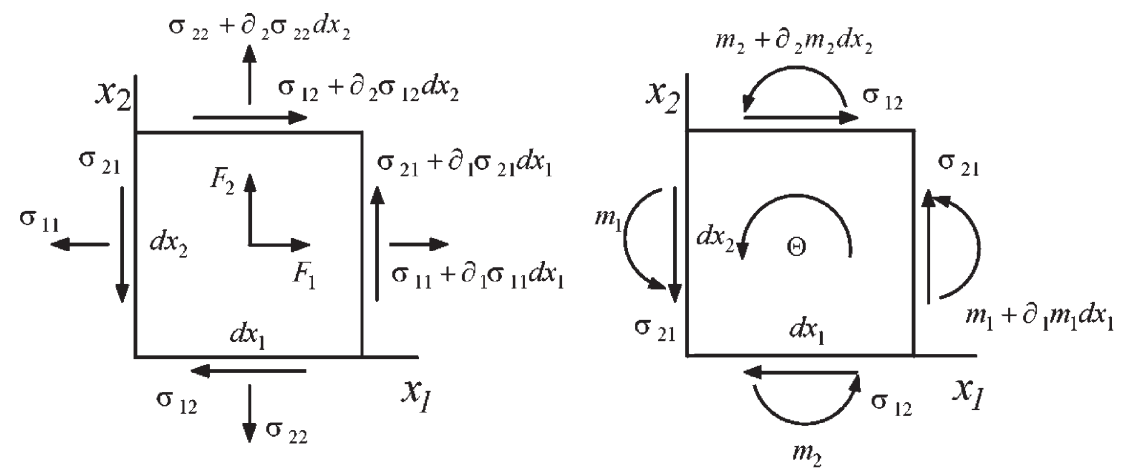

Fig. A3. Force and moment equilibrium for an infinitesimal element of Cosserat generalized continuum.

one has:

$\partial_{1} m_{1}+\partial_{2} m_{2}+\left(\sigma_{21}-\sigma_{12}\right)+\Theta=0$

where $\Theta$ is a body moment.

From Fig. A3 and the above mentioned moment equilibrium relations, we note that the shear components do not vary between a face in $x_{i}$ and its neighbor at $x_{i}+d x_{i}$. That does not mean, however, that their nonuniformity was disregarded. What happens is that the moments of the first degree terms, in the Taylor series for such components, would be infinitesimals of higher order and, therefore, would end eliminated. Physically, this can be understood as the existence of a higher gradient for couple stresses than for force stresses in the scale of an infinitesimal element.

In dynamic problems, inertia effects can be included in body forces and moments according to d'Alembert's principle (Teodorescu, 1975). Eq. (A3) shows that where non-zero couple stress gradients occur, the shear stresses $\sigma_{12}$ and $\sigma_{21}$ are not necessarily equal. One concludes therefore that the force stress tensor $\sigma_{i j}$ is, in general, nonsymmetric, similarly to the tensor of relative deformation mentioned previously.

Symmetry will occur in the static case if and only if couple stresses, although present, are self-equilibrated (body moments can generally be disregarded), or when they are identically zero as in classical continuum. In dynamic problems, the microinertial effects included in body moments in Eq. (A3) can by themselves generate the asymmetry of force stresses.

The force stresses can be split into their symmetric, $\sigma_{(i j)}=\left(\sigma_{i j}+\sigma_{j i}\right) / 2$, and anti-symmetric $\sigma_{[i j]}=\left(\sigma_{i j}-\sigma_{j i}\right) / 2$ components. Particularly, these components are denoted by $\sigma_{(12)}=\left(\sigma_{12}+\sigma_{21}\right) / 2=\sigma^{\mathrm{s}}$ and $\sigma_{[12]}=\left(\sigma_{12}-\sigma_{21}\right) / 2=\sigma^{\mathrm{a}}$. It should be noted that for the normal stress $\sigma_{(i i)} \equiv \sigma_{i i}$ and $\sigma_{[i i]} \equiv 0 . \sigma^{\mathrm{s}}$ corresponds to the classical shear stresses and provokes only distortion of the material points. $\sigma^{\mathrm{a}}$, which is related to the couple stresses by the Eq. (A3), produces exclusively rotation (Fig. A4). The understanding of the way that each component acts on the deformation of the generalized continuum, is important for formulation of strength criteria. These criteria must be specific for each failure mechanism. $\sigma^{\mathrm{s}}$ may generate sliding while $\sigma^{\mathrm{a}}$ may generate toppling (rotation).

It is remarkable that Ramsay (1967) had already noticed the 'need' of an asymmetry in the stress tensor. Even based in mechanically inconsistent concepts and apparently not aware of the generalized theory of Cosserat, that author suggested that part of the deviatoric tensor would 'not be in equilibrium', what was called by him the 'disequilibrium component' (Ramsay, 1967, p. 283). This is precisely the anti-symmetric portion of the shear stresses $\left(\sigma^{\mathrm{a}}\right)$ that, as described previously, is responsible for the asymmetry of the force stress tensor of Cosserat. Perhaps, it was not by chance that Ramsay (1967) concluded the existence of this non equilibrated component, based on his perception that rigid rotations of points of the medium (microrotations) were commonly independent of the rigid body rotations (macrorotations, $\left.\Omega_{12}=\omega_{3}=\left(\partial_{2} u_{1}-\partial_{1} u_{2}\right) / 2\right)$ and would require the definition of associated stresses. What perhaps was not fully perceived by Ramsay (1967) is that there is no disequilibrium, but the existence of couple stresses that oppose the effect of anti-symmetric shear stresses. This is exactly what is expressed by Eq. (A3).
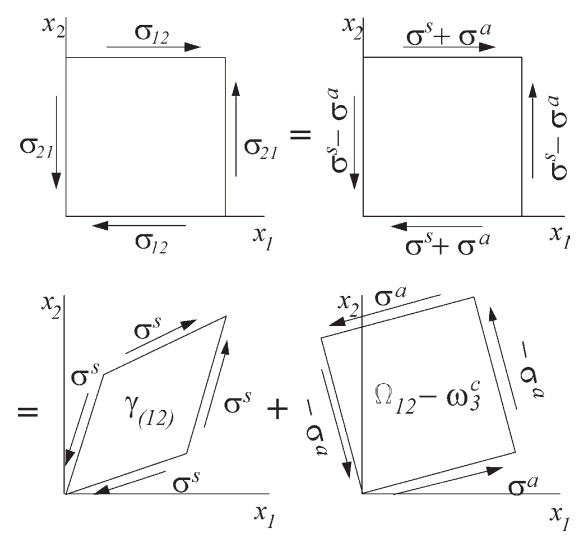

Fig. A4. Interpretation of the effect of the symmetric and anti-symmetric components of shear stresses (Mindlin, 1963). 


\section{A3. Virtual Work Principle}

In mechanics, displacements are concrete quantities, having a physical existence, while forces (and stresses) are postulated and abstract. It is therefore instructive to introduce and define the latter through the virtual work they produce under given virtual displacements. The proposition, in a general way, consists of obtaining the statics of the medium from the kinematics, through the Principle of Virtual Work (Chou and Pagano, 1967). When the kinematic description is refined, as in the case of generalized continua, it is natural that new static quantities are sought through its energetic dualities (conjugations), expressed in the Principle of Virtual Work (Germain, 1973). Used in this form, this principle constitutes a method through which it is possible to define, in a systematic way, the statics and the boundary conditions (Germain, 1973), justifying, for example, the appearance of the couple stresses in the Cosserat theory.

The application of the method starts by establishing the virtual work of the internal forces for the generalized medium. This virtual work has to be a quantity invariant with respect to rigid body motion (axiom of objectivity of the virtual work of internal forces; Germain, 1973). So the objective tensors $\gamma_{i j}$ and $\kappa_{i}$ will be used in the expression of the virtual work. Specifically for the development of the current section, we will use the tensorial (indicial) notation (Chou and Pagano, 1967) with their usual conventions such as, for example, the implicit summation for repeated subscripts (Einstein's convention). In the present work, due to the fact that only plane problems are being treated, the range of variation of the subscripts will be $i, j=1,2$. In indicial notation, the above mentioned tensors can be written as $\gamma_{i j}=\partial_{j} u_{i}+e_{i j 3} \omega_{3}^{c}$ and $\kappa_{i}=\partial_{i} \omega_{3}^{c}$, where $e_{i j 3}$ is the permutation (or alternating) tensor in 2D (Chou and Pagano, 1967). It is assumed that the medium occupies a volume $V$ with boundary $\Gamma$. In this case, the virtual work of the internal forces $\left(\delta W^{\mathrm{I}}\right)$ will be given by:

$\delta W^{\mathrm{I}}=\int_{V}\left(\sigma_{i j} \delta \gamma_{i j}+m_{i} \delta \kappa_{i}\right) \mathrm{d} V$

where the tensorial coefficients $\sigma_{i j}$ and $m_{i}$ are the introduced static variables, which are associated, in energy, with the adopted kinematics.

$\delta$ in Eq. (A4) indicates that the kinematic quantities are virtual. If kinematically admissible, i.e. satisfying the boundary conditions, these quantities do not interfere with the equilibrium (Chou and Pagano, 1967). Apart from that they are entirely arbitrary and by consequence independent. In the case of classic continuum, the only term present in the integrand would be the first. Rewriting Eq. (A4), considering the definitions of the kinematic tensors, gives:

$\delta W^{\mathrm{I}}=\int_{V}\left[\sigma_{i j}\left(\partial_{j} \delta u_{i}+e_{i j 3} \delta \omega_{3}^{c}\right)+\left(m_{i} \partial_{i} \delta \omega_{3}^{c}\right)\right] \mathrm{d} V$ and, therefore

$$
\begin{aligned}
\delta W^{\mathrm{I}} & =\int_{V}\left[\sigma_{i j} \partial_{j} \delta u_{i}+\sigma_{i j} e_{i j 3} \delta \omega_{3}^{c}+m_{i} \partial_{i} \delta \omega_{3}^{c}\right] \mathrm{d} V \\
& =\int_{V}\left[\sigma_{i j} \partial_{j} \delta u_{i}+2 \sigma^{\mathrm{a}} \delta \omega_{3}^{c}+m_{i} \partial_{i} \delta \omega_{3}^{c}\right] \mathrm{d} V
\end{aligned}
$$

where an equivalent form of the second term was used on the right hand side of the equality. This results from the definition given to $\sigma^{\mathrm{a}}$ in item $\mathrm{A} 2$ that, in indicial notation, is $\sigma^{\mathrm{a}}=\frac{1}{2} e_{i j 3} \sigma_{i j}$ (Chou and Pagano, 1967). By applying the rules of differentiation of a product to the first and third terms of the integrand of Eq. (A6), or, what is the same, integrating by parts leads to:

$$
\begin{aligned}
\delta W^{\mathrm{I}}= & \int_{V}\left\{\left[\partial_{j}\left(\sigma_{i j} \delta u_{i}\right)-\partial_{j} \sigma_{i j} \delta u_{i}\right]+2 \sigma^{\mathrm{a}} \delta \omega_{3}^{c}\right. \\
& \left.+\left[\partial_{i}\left(m_{i} \delta \omega_{3}^{c}\right)-\partial_{i} m_{i} \delta \omega_{3}^{c}\right]\right\} \mathrm{d} V
\end{aligned}
$$

By applying the Gauss-Ostrogradski divergence theorem (Chou and Pagano, 1967) to the first and fourth terms and putting $\delta \omega_{3}^{c}$ in evidence in the third and fifth terms, gives:

$$
\begin{aligned}
\delta W^{\mathrm{I}}= & -\int_{V} \partial_{j} \sigma_{i j} \delta u_{i} \mathrm{~d} V-\int_{V}\left(\partial_{i} m_{i}-2 \sigma^{\mathrm{a}}\right) \delta \omega_{3}^{c} \mathrm{~d} V \\
& +\int_{\Gamma} \sigma_{i j} \delta u_{i} n_{j} \mathrm{~d} \Gamma+\int_{\Gamma} m_{i} \delta \omega_{3}^{c} n_{i} \mathrm{~d} \Gamma,
\end{aligned}
$$

where $n_{i}$ is the unit normal vector pointed to the exterior of $\Gamma$.

The form of the expression of virtual work of the internal forces, extended to a Cosserat continuum (Eq. (A4)), motivates further extension to quantify the virtual work of the external forces $\left(\delta W^{\mathrm{E}}\right)$; for body forces $\left(\delta W_{V}^{\mathrm{E}}\right)$ and surface forces $\left(\delta W_{\Gamma}^{\mathrm{E}}\right)$ :

$\delta W_{V}^{\mathrm{E}}=\int_{V} F_{i} \delta u_{i} \mathrm{~d} V+\int_{V} \Theta \delta \omega_{3}^{c} \mathrm{~d} V$,

$\delta W_{\Gamma}^{\mathrm{E}}=\int_{\Gamma} t_{i} \delta u_{i} \mathrm{~d} \Gamma+\int_{\Gamma} m \delta \omega_{3}^{c} \mathrm{~d} \Gamma$,

$\delta W^{\mathrm{E}}=\delta W_{V}^{\mathrm{E}}+\delta W_{\Gamma}^{\mathrm{E}}$,

where $F_{i}$ is a body force, $t_{i}$ is a surface traction, $\Theta$ is a body moment, and $m$ is a surface moment. This extension of $\delta W^{\mathrm{E}}$ is due to the fact that in classical continuum, the existence of a stress tensor (a second order tensor) in the internal work leads to the appearance of force vectors (first order tensors) in the external work. By analogy, existing vector of couple stresses in the internal work, one scalar quantity (body and surface moments, zero order tensors) in the external work should appear.

The following step is the application of the virtual work principle itself. It establishes that for statically admissible fields of stresses, i.e. those that satisfy the differential equations of equilibrium (Eqs. (A2a), (A2b) and (A3)) and the natural boundary conditions, the virtual work of the 
external forces on any assumed, equally admissible, kinematic fields is equal to the work performed by the internal forces, i.e. $\delta W^{\mathrm{I}}=\delta W^{\mathrm{E}}$.

This is the direct statement of the principle. The inverse statement, which is used in the present work, establishes that, if for the static fields assumed, $\delta W^{\mathrm{I}}=\delta W^{\mathrm{E}}$, considering all admissible kinematic virtual fields, then the former (static fields) will be statically admissible. This inverse statement allows the obtaining of equilibrium equations and the natural boundary conditions as follows.

A mathematical statement of the principle $\left(\delta W^{\mathrm{I}}=\delta W^{\mathrm{E}}\right)$, through Eqs. (A8), (A9a) and (A9b), is therefore:

$$
\begin{aligned}
& -\int_{V} \partial_{j} \sigma_{i j} \delta u_{i} \mathrm{~d} V-\int_{V}\left(\partial_{i} m_{i}-2 \sigma^{\mathrm{a}}\right) \delta \omega_{3}^{c} \mathrm{~d} V \\
& \quad+\int_{\Gamma} \sigma_{i j} \delta u_{i} n_{j} d \Gamma+\int_{\Gamma} m_{i} \delta \omega_{3}^{c} n_{i} \mathrm{~d} \Gamma \\
& =\int_{V} F_{i} \delta u_{i} \mathrm{~d} V+\int_{V} \Theta \delta \omega_{3}^{c} \mathrm{~d} V \\
& \quad+\int_{\Gamma} t_{i} \delta u_{i} \mathrm{~d} \Gamma+\int_{\Gamma} m \delta \omega_{3}^{c} \mathrm{~d} \Gamma,
\end{aligned}
$$

As the variations of $u_{i}$ and $\omega_{3}^{c}$ in Eq. (A10) are arbitrary and independent, it is possible to equate directly their coefficients and transform it into two new equations; the first common to the classical continuum and the second specific to the generalized Cosserat continuum, which are:

$$
\begin{gathered}
-\int_{V} \partial_{j} \sigma_{i j} \delta u_{i} \mathrm{~d} V+\int_{\Gamma} \sigma_{i j} \delta u_{i} n_{j} \mathrm{~d} \Gamma \\
=\int_{V} F_{i} \delta u_{i} \mathrm{~d} V+\int_{\Gamma} t_{i} \delta u_{i} \mathrm{~d} \Gamma,
\end{gathered}
$$

and

$$
\begin{gathered}
-\int_{V}\left(\partial_{i} m_{i}-2 \sigma^{\mathrm{a}}\right) \delta \omega_{3}^{c} \mathrm{~d} V+\int_{\Gamma} m_{i} \delta \omega_{3}^{c} n_{i} \mathrm{~d} \Gamma \\
=\int_{V} \Theta \delta \omega_{3}^{c} \mathrm{~d} V+\int_{\Gamma} m \delta \omega_{3}^{c} \mathrm{~d} \Gamma
\end{gathered}
$$

By reorganizing the terms, we obtain:

$$
\int_{V}\left\{\left(\partial_{j} \sigma_{i j}+F_{i}\right) \delta u_{i}\right\} \mathrm{d} V+\int_{\Gamma}\left[\left(t_{i}-\sigma_{i j} n_{j}\right) \delta u_{i}\right] \mathrm{d} \Gamma=0(\mathrm{~A} 12 \mathrm{a})
$$

and

$$
\begin{array}{r}
\int_{V}\left\{\left(\partial_{i} m_{i}-2 \sigma^{\mathrm{a}}+\Theta\right) \delta \omega_{3}^{c}\right\} \mathrm{d} V \\
+\int_{\Gamma}\left[\left(m-m_{i} n_{i}\right)\right] \delta \omega_{3}^{c} \mathrm{~d} \Gamma=0
\end{array}
$$

These equations will only be satisfied for arbitrary variations if, simultaneously in each one of them, the integrands of the volume and surface integrals are zero. So the equilibrium equations

$\partial_{j} \sigma_{i j}+F_{i}=0$,

and

$\partial_{i} m_{i}-2 \sigma^{a}+\Theta=0$,

are obtained, which are equivalent to Eqs. (A2a), (A2b) and (A3) written in indicial notation, and the natural boundary conditions

$t_{i}=\sigma_{i j} n_{j}\left(\right.$ Cauchy stress equation applied to $\left.\Gamma_{\sigma}\right)$

and

$m=m_{i} n_{i}$.

To evaluate the integrals in the equations above, it is necessary to assume that boundary $\Gamma$ is defined in complementary parts $\Gamma_{u}, \Gamma_{\omega}, \Gamma_{\sigma}$ and $\Gamma_{m}$. The natural boundary conditions in Eqs. (A14a) and (A14b) are prescribed on $\Gamma_{\sigma}$ and $\Gamma_{m}$, respectively. The essential boundary conditions related to $u_{i}$ and $\omega_{3}^{c}$ are prescribed on $\Gamma_{u}$ and $\Gamma_{\omega}$, respectively.

\section{A4. Stress-strain relationships}

The equations for the linear elastic isotropic behaviour of a 2D Cosserat continuum involve four parameters. They can be written as (Teodorescu, 1975):

$\lambda, G, G_{c}$ and $B=2 G l^{2}$,

where $\lambda$ and $G$ are analogous to Lame's classical parameters (Jaeger and Cook, 1979), $G_{c}$ is an anti-symmetric (or rotational) shear modulus, $B$ is a flexural modulus (having dimension of force) and $l$ is the characteristic length of the medium.

The stress-strain relations is stated as:

$\sigma_{i j}=\lambda \epsilon_{k k} \delta_{i j}+\left(G+G_{c}\right) \gamma_{i j}+\left(G-G_{c}\right) \gamma_{j i}$,

and

$m_{3 i}=m_{i}=B \kappa_{i}$.

Eq. (A15a) can be rewritten as:

$\sigma_{i j}=\lambda \epsilon_{k k} \delta_{i j}+2 G \epsilon_{i j}+2 G_{c} \gamma_{[i j]}$,

in function of the symmetric and anti-symmetric parts of the relative deformations defined in item A1 of this Appendix. It can be noted that two new parameters appear: $G_{c}$, which relates the anti-symmetric parts of the force stresses and the relative strains and $l$, which introduces flexural effects (and by consequence scale effects) in the medium, indirectly measuring the dimensions of its internal structure. In this case, where the material is isotropic, only one characteristic length exists.

Additional strength criteria must also be introduced for the definition of the Cosserat generalized continuum. These 
strength criteria represent the additional failure mechanisms induced by the anti-symmetric force stresses and by couple stresses. For the analysis of the bookshelf mechanism focused on in this paper, a criterion representing failure by rotation was given by Eq. (1).

\section{Appendix B. The 2D Mohr circle for Cosserat continuum}

In the generalized Cosserat continuum, like in the classical continuum, the force stresses in 2D can be represented graphically by a circle in the Mohr stress space, i.e. the space of normal stresses versus shear stresses $\left(\sigma_{\mathrm{N}} \times \sigma_{\mathrm{S}}\right)$. This type of representation is derived in the present Appendix.

Fig. A5 shows a free body diagram where the components of force stresses are indicated. The faces of the diagram are oriented perpendicular to the coordinate axes and to a unit vector $n_{i}$ making an angle $\alpha$ (arbitrary) to the $x$ axis.This unit vector has components $\left(n_{1}, n_{2}\right)^{t} \equiv(\cos \alpha, \operatorname{sen} \alpha)^{t}$, while the components of a vector $s_{i}$, parallel to the plane, are $\left(s_{1}, s_{2}\right)^{t} \equiv(-\operatorname{sen} \alpha, \cos \alpha)^{t}$.

By imposing an equilibrium of forces along the coordinate axes, it is possible to determine the respective components of the vector of force stresses, $t_{i}$, in the plane normal to $n_{i}$ (hereafter designated by plane $\alpha$ ), knowing that $t_{i}=\sigma_{i j} n_{j}$. The projection of $t_{i}$ over $n_{i}$ and $s_{i}$ will give, respectively, the normal $\left(\sigma_{\mathrm{N}}\right)$ and shear $\left(\sigma_{\mathrm{S}}\right)$ stress components in plane $\alpha$ as:

$\sigma_{\mathrm{N}}=t_{i} n_{i}=\sigma_{i j} n_{j} n_{i}$

$\sigma_{\mathrm{S}}=t_{i} s_{i}=\sigma_{i j} n_{j} s_{i}$

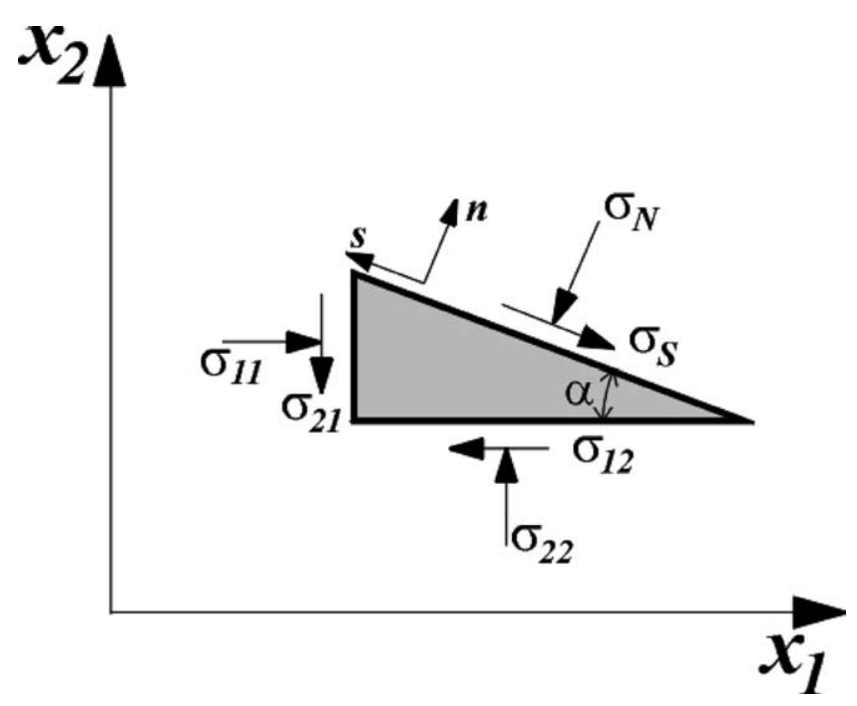

Fig. A5. Free body diagram for a 2D state of stress of an infinitesimal element.
These expressions, in extended form, are:

$$
\begin{aligned}
\sigma_{\mathrm{N}}= & \sigma_{11} \cos ^{2} \alpha+\sigma_{22} \operatorname{sen}^{2} \alpha \\
& +\left(\sigma_{12}+\sigma_{21}\right) \operatorname{sen} \alpha \cos \alpha, \\
\sigma_{\mathrm{S}}= & \sigma_{21} \cos ^{2} \alpha-\sigma_{12} \operatorname{sen}^{2} \alpha \\
& +\left(\sigma_{22}-\sigma_{11}\right) \operatorname{sen} \alpha \cos \alpha,
\end{aligned}
$$

or, in terms of $2 \alpha$ :

$$
\begin{aligned}
& \sigma_{\mathrm{N}}-\frac{1}{2}\left(\sigma_{11}+\sigma_{22}\right) \\
& \quad=\frac{1}{2}\left(\sigma_{11}-\sigma_{22}\right) \cos 2 \alpha+\frac{1}{2}\left(\sigma_{12}+\sigma_{21}\right) \operatorname{sen} 2 \alpha,
\end{aligned}
$$

$$
\begin{aligned}
\sigma_{\mathrm{S}} & -\frac{1}{2}\left(\sigma_{21}-\sigma_{12}\right) \\
& =\frac{1}{2}\left(\sigma_{22}-\sigma_{11}\right) \operatorname{sen} 2 \alpha+\frac{1}{2}\left(\sigma_{12}+\sigma_{21}\right) \cos 2 \alpha .
\end{aligned}
$$

Squaring both sides and adding Eqs. (A17c) and (A17d) we arrive at the equation of a circle in the plane $\left(\sigma_{\mathrm{S}}, \sigma_{\mathrm{N}}\right)$ :

$$
\begin{aligned}
& \left(\sigma_{\mathrm{N}}-\sigma_{m}\right)^{2}+\left(\sigma_{\mathrm{S}}+\sigma^{a}\right)^{2} \\
& \quad=\left(\frac{\sigma_{11}-\sigma_{22}}{2}\right)^{2}+\left(\frac{\sigma_{12}+\sigma_{21}}{2}\right)^{2},
\end{aligned}
$$

where $\sigma_{m}=\left(\sigma_{11}+\sigma_{22}\right) / 2$ and $\sigma^{\mathrm{a}}=\left(\sigma_{12}-\sigma_{21}\right) / 2$ are, respectively, the average normal stress and the antisymmetric shear stress. Therefore the locus of the states of force stresses in $2 \mathrm{D}$ represented on plane is a circle, as in the classical continuum. The difference is that its center undergoes a translation $-\sigma^{\mathrm{a}}$ in a direction perpendicular to the axis of normal stresses, being therefore located in the point of coordinates $\left(\sigma_{m},-\sigma^{\mathrm{a}}\right)$.

Fig. A6 shows a Mohr circle for the state of stress represented in Fig. A5. In the adopted convention, the tensile stresses are positive. Therefore, the shear stresses (symmetric and anti-symmetric) are plotted as positive when producing clockwise rotating couples, i.e. dextral shear in geological nomenclature (see Chou and Pagano, 1967, section 1.5).

All the elegant graphical solutions of structural geology problems obtained with the Mohr circle in the classical continuum (Mandl, 2000), are possible in the Cosserat continuum with minimal adaptations. Values and orientations of principal stresses, which may not be mutually perpendicular and may not even exist, if the value of $\sigma^{\mathrm{a}}$ is such that the circle does not intercept the $\sigma_{\mathrm{N}}$ axis, can be determined by procedures as those used in classical continuum. The same applies to the determination of maximum and minimum normal stresses (which no longer coincide with the principal stresses) and to the calculation of the maximum shear stress. The concept of pole, or origin of planes (point P in Fig. A6), is equally valid in the graphical construction. 


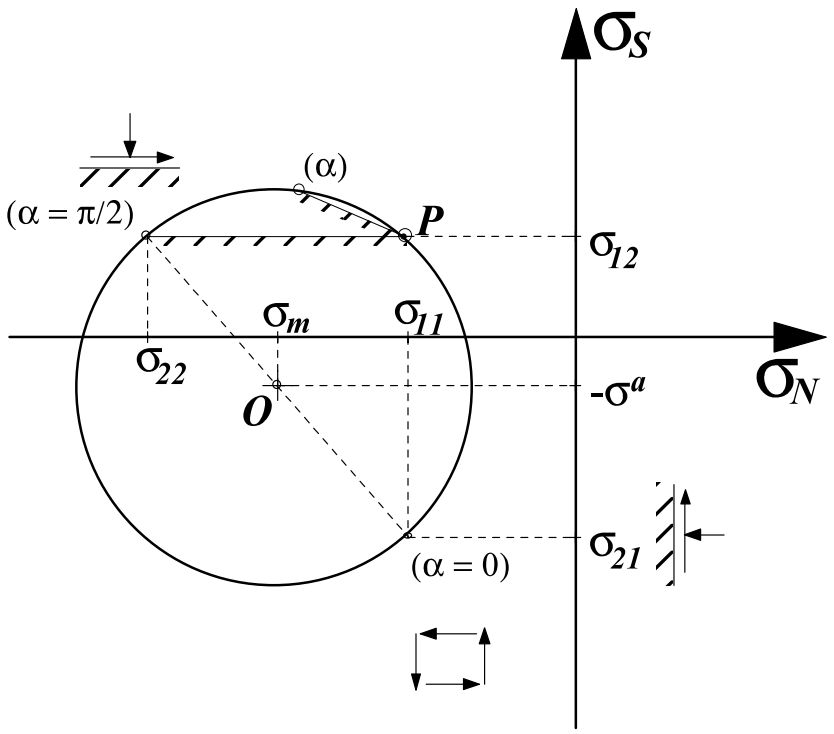

Fig. A6. Mohr circle for a 2D Cosserat generalized continuum.

Fig. A7 shows the effect of the anti-symmetric part of the shear stresses in inducing failure in a point of the medium. Through the displacement in a direction perpendicular to the normal stress axis, the circle may become tangent to one strength envelope without increasing its diameter. This corresponds to an exclusive increase of the anti-symmetric part of the stresses, which, as mentioned before, is related to rotations (as occurs in the bookshelf mechanism).

For three-dimensional states of stresses, the graphical representation cannot be given by a combination of three circles in the plane $\sigma_{\mathrm{S}} \times \sigma_{\mathrm{N}}$ as in classical continuum (e.g. Jaeger and Cook, 1979). The representation for the total (non-symmetric) stress tensor (Unterreiner, 1995) is given by more complex curves (planar sections of quadric surfaces), which reduces its applicability.

Nikolaevski (1996) introduced the couple stresses, together with force stresses, in the equilibrium of moments in the free-body diagram of Fig. A5. By doing so, the

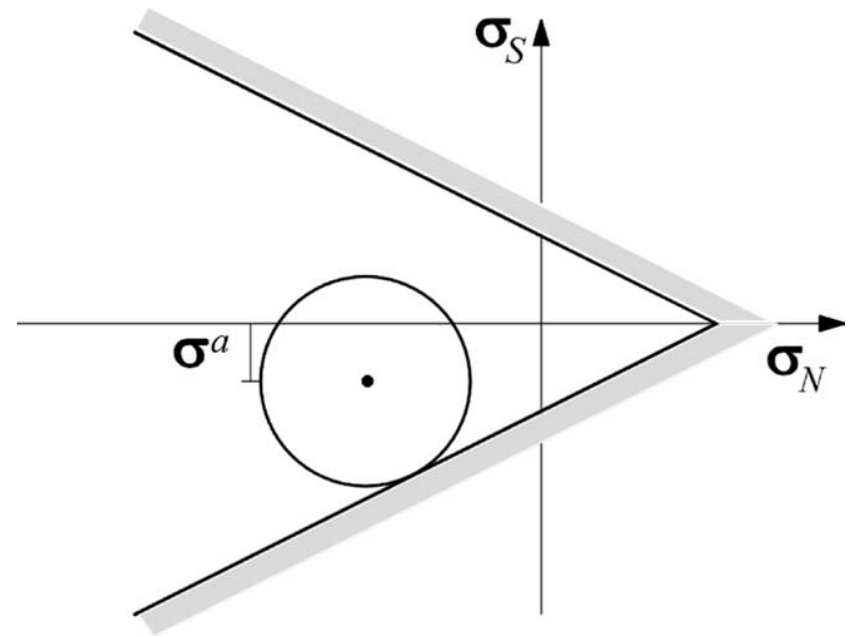

Fig. A7. Failure produced by anti-symmetric stresses. pertinent space of stresses becomes three-dimensional $\left(\sigma_{\mathrm{S}} \times \sigma_{\mathrm{N}} \times m\right)$ and its projection on a plane takes to an elliptical form for the graphical representation of the $2 \mathrm{D}$ stress tensor. In the authors' opinion, the implications of this form, as well as of the possible variants of Cosserat Mohr circles, in the analysis of geological structures, deserves appreciation.

Some works (e.g. Lister and Williams, 1983; Means, 1983; De Paor and Means, 1984; Bobyarchick, 1986) involve the use of Mohr circles that may not be centered in the abscissa axis. These are circles for displacement or velocity gradients, which are non-symmetric second order tensors, similar to the force stress tensor in the Cosserat theory. These circles have been described by Prager (1961) and are not the same as the Mohr circles for strain quantities of Cosserat continua, described in item A1. In the Cosserat mechanics, rotations are independent of displacements. In contrast, for the above mentioned displacement gradients, rotations are their anti-symmetric portion and, as a consequence, dependent on them. These circles have been utilized in the kinematic analysis of shear zones (Simpson and De Paor, 1993). They are not able, however, to take into account the independence of the rotations in the interior of the shear zones in relation to rotations in the remaining medium (Mühlhaus and Vardoulakis, 1987). This is only possible by using Cosserat Mohr circles for strain.

\section{Appendix C. A strength criterion for rolling friction: toppling of blocks}

Consider rectangular blocks $\mathrm{ABCD}$, with height $h$, width $b$ and unit thickness perpendicular to their plane, as illustrated in Fig. A8. Assume that a normal stress $\sigma_{\mathrm{N}}$ and

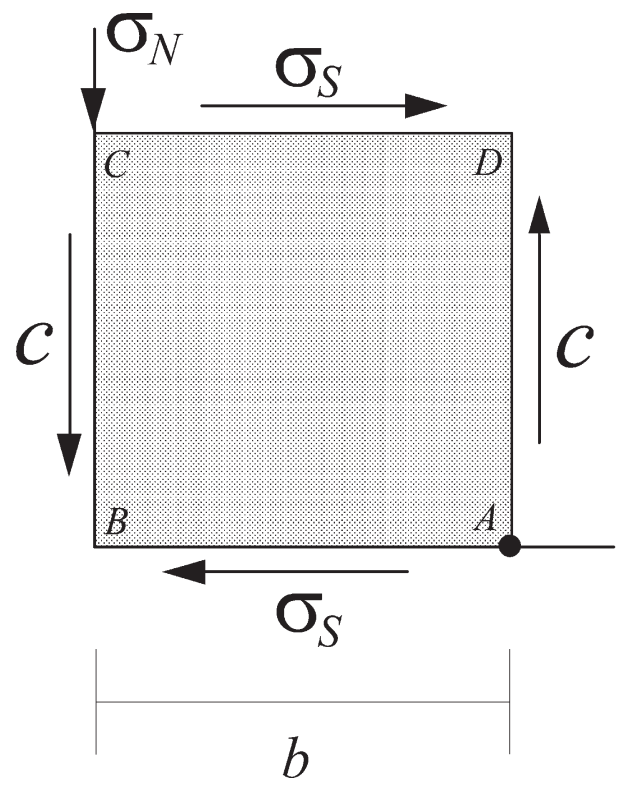

Fig. A8. Moment equilibrium for a block considering a toppling failure criterion. 
a shear stress $\sigma_{\mathrm{S}}$ act on the horizontal planes, according to Fig. A8, and that there is cohesion available in all vertical planes, between adjacent blocks. The moment equilibrium for a given block in the medium, relative to the point $\mathrm{A}$, is:

$\sum M_{\mathrm{A}}=\left(\sigma_{\mathrm{S}} b\right) h-(c h) b-\left(\sigma_{\mathrm{N}} b\right) b=0$,

where $\Sigma M_{\mathrm{A}}$ is the summation of non zero moments of the various forces in relation to point A. Simplifying the above expression gives:

$\sigma_{\mathrm{S}}=\sigma_{\mathrm{N}}(b / h)+c$.

By calling $b / h=\tan \varphi$, we have:

$\sigma_{\mathrm{S}}=\sigma_{\mathrm{N}} \tan \varphi+c$,

where $\varphi$ denotes an angle representative of the slenderness of the blocks and is defined as the rolling friction angle. Eq. (A19) represents the required shear stress for the blocks to topple (roll), and is analogous to Eq. (1) with a cohesion term included.

\section{References}

Amadei, B., Stephansson, O., 1997. Rock Stress and its Measurement, Chapman and Hall, London.

Besdo, D., 1985. Inelastic behavior of plane frictionless block-systems described as Cosserat media. Archive of Mechanic 37, 603-619.

Biot, M.A., 1967. Rheological stability with couple-stresses and its applications to geological folding. Proceedings Royal Society of London A2298, 402-423.

Bobyarchick, A.R., 1986. The eigenvalues of steady flow in Mohr space. Tectonophysics 122, 35-51.

Cauchy, A.L., 1850. Mémoire sur les systèmes isotropes de points matériels. Mémoire de l'Académie des Sciences, Paris, 22.

Chappell, B.A., 1979. Load distribution and redistribution in discontinua. International Journal of Rock Mechanics and Mining Sciences and Geomechanics Abstracts 12, 265-270.

Chou, P.C., Pagano, N., 1967. Elasticity: Tensor, Dyadic and Engineering Approaches, Dover Publications, New York.

Cosserat, E., Cosserat, F., 1909. Théorie des Corps Déformables, Hermann et Fils, Paris.

De Paor, D.G., Means, W.D., 1984. Mohr circles of the first and second kind and their use to represent tensor operations. Journal of Structural Geology 6, 693-701.

Fadeev, A.B., Kuzevanov, V.V., 1993. Strength of regularly jointed media. In: Pasamehmetoglu, A.G., et al. (Eds.), Proceedings of Assessment and Prevention of Failure Phenomena in Rock Engineering, Balkema, Rotterdam, pp. 175-179.

Figueiredo, R.P., 1999. Rock mass modelling as Cosserat generalized continuous media (in Portuguese). PhD. thesis, Civil Engineering Department, Pontifícia Universidade Católica do Rio de Janeiro, Rio de Janeiro, Brazil.

Figueiredo, R.P., Vargas, E.A., 2001. Sigmoidal veins: a model of its geometry/origin based on the Cosserat generalized continuum mechanics (in Portuguese). In: SBG-Brazilian Geological Society (Eds.), Proceedings of the Second International Symposium on Tectonics of the Brazilian Geological Society, Recife, Brazil, pp. 205-208.

Germain, P., 1973. The method of virtual power in continuum mechanicspart 2: microstructure. SIAM Journal of Applied Mathematics 25, $556-575$.

Goodman, R.E., 1976. Methods of Geological Engineering in Discontinuous Rocks, West Publishing, St. Paul.
Jaeger, J.C., Cook, N.G.W., 1979. Fundamentals of Rock Mechanics, 3rd ed, Chapman and Hall, London.

Kröner, E. (Ed.), 1968. Mechanics of Generalized Continua. Proceedings of IUTAM Symposium, International Union of Theoretical and Applied Mechanics, Springer-Verlag, Berlin.

Latham, J.P., 1985a. The influence of nonlinear materials properties and resistance to bending on the development of internal structures. Journal of Structural Geology 7, 225-236.

Latham, J.P., 1985b. A numerical investigation and geological discussion of the relationship between folding, kinking and fracturing. Journal of Structural Geology 7, 237-249.

Lippmann, H., 1969. Eine Cosserat-Theorie das plastischen Flie(ens. Acta Mechanica 8, 255-284.

Lister, G.S., Williams, P.F., 1983. The partitioning of deformation in flowing rock masses. Tectonophysics 92, 1-33.

Mandl, G., 1987. Tectonic deformation of rotating parallel faults-the 'bookshelf' mechanism. Tectonophysics 141, 277-316.

Mandl, G., 1988. Mechanics of Tectonic Faulting, Elsevier, Amsterdam.

Mandl, G., 2000. Faulting in Brittle Rocks-An Introduction to the Mechanics of Tectonic Faults, Springer-Verlag, Berlin.

Mase, G.E., Mase, G.H., 1992. Continuum Mechanics for Engineers, CRC Press, Boca Raton.

Mattauer, M., 1973. Les Déformations des Matériaux de l'Écorce Terrestre, Hermann et Fils, Paris.

Means, W.D., 1983. Application of Mohr-circle construction to problems of inhomogeneous deformation. Journal of Structural Geology 5, 279-286.

Mindlin, R.D., 1963. Influence of couple-stresses on stress concentrations. Experimental Mechanics 3, 1-7.

Mühlhaus, H.-B., Vardoulakis, J., 1987. The thickness of shear bands in granular materials. Géotechnique 37, 271-283.

Nascimento, U., Teixeira, H., 1971. Mechanisms of internal friction in soils and rocks. In: ISRM (Eds.), Proceedings of ISRM Symposium, Nancy, France, p. II-3.

Nikolaevski, V.N., 1996. Geomechanics and Fluidodynamics-with Applications to Reservoir Engineering, Kluwer Academic Publishers, Dordrecht, The Netherlands.

Poisson, S.D., 1842. Mémoire sur l'équilibre et le mouvement des corps cristallisés. Mémoires de l'Académie des Sciences, Paris, 18

Prager, W., 1961. Introduction to Mechanics of Continua, Ginn and Company, Boston.

Price, N.J., Cosgrove, J.W., 1990. Analysis of Geological Structures, Cambridge University Press, Cambridge.

Ramsay, J.G., 1967. Folding and Fracturing of Rocks, McGraw-Hill, New York.

Ramsay, J.G., Huber, M., 1987. The Techniques of Modern Structural Geology_Volume 2: Folds and Fractures, Academic Press, London.

Saint Venant, A.J.C.B., 1869. Note sur les valeurs que prennent les pressions dans un solide élastique isotrope lorsque l'on tient compte des dérivées d'ordre supérieur des déplacements très petits que leurs points ont éprouvés. Compte Rendus Hebdomadaires des Séances de l'Académie des Sciences, Paris, 68.

Sheorey, P.R., 1994. A theory for in situ stresses in isotropic and transversely isotropic rock. International Journal of Rock Mechanics and Mining Sciences and Geomechanics Abstracts 31, 23-34.

Simpson, C., De Paor, D.G., 1993. Strain and kinematic analysis in general shear zones. Journal of Structural Geology 15, 1-20.

Teodorescu, P.P., 1975. Dynamics of Linear Elastic Bodies, Editura Academiei, Bucarest, Rumania.

Unterreiner, P., 1995. Contribution à l'étude et à la modélisation numérique des sols cloués: application au calcul en déformation des ouvrages de soutènement. These de Doctorat, École Nationale des Ponts et Chaussées, Paris, France.

Voigt, W., 1887. Theoretische Studien über die Elastizitätsverhältnisse der Krystalle, I, II. Abhandlungen der Königlichen Gesellschaft der Wissenschaften zu Göttingen, 34. 